\title{
LA PRÁCTICA CONVENCIONAL DE ESPAÑA Y FILIPINAS RELATIVA A LOS TRATADOS SOBRE DERECHOS HUMANOS EN MATERIA DE RESERVAS*
}

\author{
The Conventional Practice of Spain and The Philippines \\ concerning reservations to Human Rights Treaties
}

\author{
Alicia María Pastor-García \\ Universidad de Málaga \\ ampastor@uma.es \\ doi: http://dx.doi.org/10.18543/ed-66(1)-2018pp303-316
}

Recibido: 12.04 .2018

Aceptado: 25.06.2018

\section{Resumen}

El presente artículo se centra en el análisis de las reservas de España y Filipinas a distintas convenciones internacionales que tienen como finalidad la protección efectiva de los Derechos Humanos. De esta manera, hemos considerado que el estudio de las reservas hechas a un elevado número de convenciones multilaterales destinadas a la protección de los Derechos Humanos, así como las objeciones formuladas a las mismas, representa una forma idónea de constatar la práctica de España y Filipinas en la salvaguardia de estos derechos.

\section{Palabras clave}

Reservas, Derechos Humanos, Convenciones, España. Filipinas.

* Cómo citar / Citation 'Chicago-Deusto' (Autor-fecha / Author-date / Lista de referencias / Reference list entries): Pastor García, Alicia María. 2018. «La práctica convencional de España y Filipinas relativa a los tratados sobre Derechos Humanos en materia de reservas». Estudios de Deusto 66, n. ${ }^{\circ}$ 1: 303-316. doi: http://dx.doi.org/10.18543/ed66(1)-2018pp303-316. 


\section{Abstract}

The present study focuses on analyzing the reservations of Spain and the Philippines to different international conventions that aim to effectively protect Human Rights. In this sense, we have considered that the analysis of the reservations, as well as the objections made to them, made to a large number of multilateral conventions destined to the protection of Human Rights represents a suitable way to verify the practice of Spain and the Philippines towards safeguarding these rights.

\section{Keywords}

Reservations, Human Rights, conventions, Spain, the Philippines. 
Sumario: I. Consideraciones Generales. II. PrÁcticA CONVEnCIONAL DE ESPAÑA Y FILIPINAS. 1. Práctica relativa a los tratados sobre Derechos Humanos. 2. Práctica relativa a los protocolos facultativos. III. REFLEXIONES FINALES.

\section{CONSIDERACIONES GENERALES}

La protección de los Derechos Humanos representa en la actualidad una de las funciones principales del Derecho Internacional, cuyo desarrollo se lleva a cabo esencialmente a través de instrumentos convencionales de carácter multilateral. No obstante, no resulta extraño en la práctica que estos convenios vean limitado su alcance por medio de las reservas ${ }^{1}$ manifestadas por los Estados Partes a los mismos, aunque esto facilite su ámbito espacial de aplicación. Esta práctica da lugar a las tensiones surgidas en torno al binomio universalidad-integridad de los convenios cuyo objeto es la protección de Derechos Humanos.

En este sentido cabe señalar que, mientras que algunos Estados optan por no ratificar aquellos tratados cuyo objeto es la protección de los Derechos Humanos, otros Estados deciden ser partes de los mismos haciendo uso del mecanismo jurídico de las reservas ${ }^{2}$. Una práctica que podría limitar el ámbito de aplicación material del tratado, en la medida en que el consentimiento de un Estado en obligarse por una convención de estas características se encontraría condicionado por el establecimiento de ciertas reservas, las cuales podrían, llegado el caso, comprometer el principio de integridad del convenio y su alcance jurídico.

El presente estudio se centra en el análisis de las reservas de España y Filipinas a distintas convenciones internacionales que tienen como finalidad la protección efectiva de los Derechos Humanos. De esta manera, hemos considerado que el análisis de las reservas hechas a un elevado número de convenciones multilaterales destinadas a la protección de los Derechos Humanos representa una forma idónea de constatar la posición de España y Filipinas hacia la salvaguardia de estos derechos, así como el examen de las objeciones formuladas a reservas de otros países.

${ }^{1}$ Definidas en el Convenio de Viena sobre el Derecho de los Tratados, cuyo artículo 2.1.d. establece que «Se entiende por «reserva» una declaración unilateral, cualquiera que sea su enunciado o denominación, hecha por un Estado al firmar, ratificar, aceptar o aprobar un Tratado o al adherirse a él, con objeto de excluir o modificar los efectos jurídicos de ciertas disposiciones del Tratado en su aplicación a ese Estado». Convenio adoptado en Viena el 23 de mayo de 1969, publicado en el BOE de 13 de junio de 1980.

2 En este sentido, vid., por todos: Horn, 1988: 514; Imbert 1979: 503 y Riquelme Cortado 2004: 434. 
Por lo que se refiere al ámbito espacial de estos tratados hemos de destacar que cuentan, en su mayoría, con una amplia ratificación. No obstante, también se han formulado un considerable número de reservas, algunas de las cuales podrían considerarse contrarias al objeto y fin del tratado, con el perjuicio resultante para la debida protección de los Derechos Humanos contenidos en estos instrumentos convencionales. Por este motivo, entendemos que la práctica de España y Filipinas en este contexto representa un buen elemento para valorar el compromiso de ambos Estados respecto de la protección de los Derechos Humanos.

Así, dentro de los tratados con un mayor número de ratificaciones, destaca la Convención sobre los Derechos del niño, adoptada por la Asamblea General de Naciones Unidas en 1989, con un total de 196 Estados partes ${ }^{3}$. Igualmente, encontramos otros muchos convenios con un alcance significativo, como es el caso de la Convención sobre la eliminación de todas las formas de discriminación contra la mujer, de 1979, de la que actualmente son partes 189 Estados; del Convenio Internacional sobre Eliminación de todas las formas de Discriminación Racial, de 1966, con un total de 178; o de la Convención sobre los derechos de las personas con discapacidad, de 2006 con 174 Estados partes. Pues bien, todos estos convenios ${ }^{5}$ cuentan con un elevado número de reservas y declaraciones interpretativas ${ }^{6}$.

Igualmente, dentro de los tratados a los que se han formulado un mayor número de reservas también figuran: el Pacto internacional de derechos civiles y políticos ${ }^{7}$; el Pacto Internacional de derechos económicos, sociales y culturales $^{8}$ y la Convención contra la tortura y otros tratos o penas crueles, inhumanos o degradantes 9 .

Por su parte, entre los tratados internacionales con un menor número de ratificaciones se encuentran: la Convención Internacional sobre la protección de los derechos de todos los trabajadores migratorios y de sus

${ }^{3}$ Estos datos, al igual que los demás, disponibles en: https://treaties.un.org/Pages/ Treaties.aspx id $=4 \&$ subid $=$ A\&clang=_en

${ }^{4}$ Se trata de la convención de Naciones Unidas que mayor número de signatarios tuvo el día de su apertura a firma. Asimismo, fue el primer convenio de Derechos Humanos en abrirse a la firma de organizaciones regionales de integración. Vid, http://www. un.org/spanish/disabilities/default.asp?id=497

Tanto es así, que la Unión Europea es parte de este tratado, con la formulación de una amplia declaración y de una reserva.

5 De todos ellos son partes España y Filipinas.

${ }^{6}$ Declaraciones que en muchos casos esconden verdaderas reservas bajo esta denominación.

7 Con un total de 169 Estados partes.

${ }^{8}$ Que cuenta en la actualidad con 165 Estados partes.

9 Con 162 Estados partes. 
familiares $^{10}$, la Convención sobre la imprescriptibilidad de los crímenes de guerra y de los crímenes de lesa humanidad ${ }^{11}$, la Convención Internacional para la protección de todas las personas contra las desapariciones forzadas ${ }^{12}$, y la Convención Internacional sobre la Represión y el Castigo del Crimen de Apartheid ${ }^{13}$. En efecto, todas ellas con un número de reservas bastante inferior al de aquellas convenciones que cuentan con un mayor alcance espacial. Si bien cabe hacer especial referencia en este contexto al Convenio constitutivo del fondo para el desarrollo de los pueblos indígenas de América Latina y el Caribe ${ }^{14}$ y a la Convención internacional contra el apartheid en los deportes ${ }^{15}$, a las que se ha formulado apenas una declaración, respectivamente ${ }^{16}$.

A la luz de estos datos cabe afirmar que el mayor alcance de los tratados puede traer aparejado un mayor número de reservas. Tanto es así, que los tratados enumerados con carácter previo cuentan con más reservas y declaraciones interpretativas que aquellos tratados que tienen un menor alcance internacional. Unos datos que evidencian la relación directamente proporcional existente entre el número de reservas y la universalidad de los convenios multilaterales.

\section{PRÁCTICA CONVENCIONAL DE ESPAÑA Y FILIPINAS}

\section{Práctica relativa a los tratados sobre derechos humanos}

Con el objetivo de constatar la posición de España y Filipinas hacia la protección de los Derechos Humanos, nuestra atención se ha dirigido a la práctica convencional que han generado ambos Estados tanto sobre las reservas como las objeciones a las mismas. En este sentido, la práctica convencional de Filipinas es bastante uniforme, ya que este Estado es parte de la práctica totalidad de los tratados que han sido analizados. No obstante, a pesar de su alta participación en los convenios internacionales cuyo objeto es

${ }^{10}$ De la que España no es parte y que cuenta con 51 Estados partes.

${ }_{11}$ De la que son partes 55 Estados, entre los que no se encuentra España.

${ }_{12}$ De la que Filipinas no es parte y que tiene 57 Estados partes.

13 Con un número de Estados partes sensiblemente mayor al de los anteriores, en concreto 109, entre los que no está España.

${ }_{14}$ Del que Filipinas no es parte.

15 De la que España no es parte.

${ }^{16}$ El Convenio constitutivo del fondo para el desarrollo de los pueblos indígenas de América Latina y el Caribe consta de 23 Estados partes y una declaración formulada por la República Bolivariana de Venezuela. Por su parte, la Convención internacional contra el apartheid en los deportes, a pesar de contar con un mayor alcance, en concreto con 60 Estados partes, sólo tiene una declaración formulada por la República de Cuba. 
la protección de Derechos Humanos, hemos de decir que la formulación de reservas por parte de este Estado es muy escasa.

Efectivamente, en el estudio llevado a cabo sólo se ha observado una reserva formulada por parte de este país, en concreto al artículo IV del Convenio para la prevención y la sanción del delito de genocidio ${ }^{17}$, relativo al castigo del delito de genocidio cometido por cualquier persona, incluidos los gobernantes. En este caso, el Gobierno de Filipinas establece que no puede sancionar ninguna acción que someta a su Jefe de Estado, de manera que queden anuladas ciertas inmunidades garantizadas por la Constitución de Filipinas. Asimismo, esta reserva hace referencia al artículo VII, cuyo efecto queda subordinado a que la legislación de este Estado establezca la definición y sanción del delito de genocidio, así como a los artículos VI y IX del citado convenio, con el objetivo de no privar de su competencia a los juzgados y tribunales de Filipinas para aquellos casos de genocidio cometidos en su territorio:

«1. With reference to article IV of the Convention, the Philippine Government cannot sanction any situation which would subject its Head of State, who is not a ruler, to conditions less favorable than those accorded other Heads of State, whether constitutionally responsible rulers or not. The Philippine Government does not consider said article, therefore, as overriding the existing immunities from judicial processes guaranteed certain public officials by the Constitution of the Philippines.

2. With reference to article VII of the Convention, the Philippine Government does not undertake to give effect to said article until the Congress of the Philippines has enacted the necessary legislation defining and punishing the crime of genocide, which legislation, under the Constitution of the Philippines, cannot have any retroactive effect.

3. With reference to articles VI and IX of the Convention, the Philippine Government takes the position that nothing contained in said articles shall be construed as depriving Philippine courts of jurisdiction over all cases of genocide committed within Philippine territory save only in those cases where the Philippine Government consents to have the decision of the Philippine courts reviewed by either of the international tribunals referred to in said articles. With further reference to article IX of the Convention, the Philippine Government does not consider said article to extend the concept of State responsibility beyond that recognized by the generally accepted principles of international law» ${ }^{18}$.

Por lo que respecta a España, también es parte de un número significativo de tratados multilaterales cuyo objeto es la protección de Derechos Humanos.

${ }^{17}$ Una convención de la que son partes un total de 147 Estados.

${ }_{18}$ El texto de esta reserva, al igual que el de las restantes reservas citadas en el presente documento, se encuentra disponible en https://treaties.un.org/Pages/ViewDetails. aspx?src=TREATY\&mtdsg_no $=I V-1 \&$ chapter $=4 \&$ clang $=$ en\#EndDec 
De hecho, este Estado ha formulado determinadas reservas y declaraciones a algunos de ellos ${ }^{19}$, aunque las mismas no representan un número muy elevado. En concreto, España formuló la siguiente reserva a la Convención sobre la eliminación de todas las formas de discriminación contra la mujer:

«La ratificación de la Convención por España no afectará a las disposiciones constitucionales en materia de sucesión a la Corona española» ${ }^{20}$.

Además hizo las siguientes declaraciones a la Convención sobre los Derechos del niño:

«1. Con respecto al párrafo d) del artículo 21 de la Convención, España entiende que de la interpretación del mismo nunca podrán deducirse beneficios financieros distintos de aquellos que fueran precisos para cubrir los gastos estrictamente necesarios que puedan derivarse de la adopción en el supuesto de niños y niñas que residan en otro país.

2. España, deseando hacerse solidaria con aquellos Estados y organizaciones humanitarias que han manifestado su disconformidad con el contenido de los párrafos 2 y 3 del artículo 38 de la Convención, quiere expresar asimismo su disconformidad con el límite de edad fijado en ellos y manifestar que el mismo le parece insuficiente, al permitir el reclutamiento y participación en conflictos armados de niños y niñas a partir de los quince años $\rangle^{21}$.

Por otro lado, resulta igualmente interesante tratar de evaluar la posición de España y Filipinas en lo que se refiere a la formulación de objeciones a aquellas reservas que consideran incompatibles con el objeto y finalidad del convenio. No obstante, cabe decir que es habitual que las objeciones establezcan que las mismas no impiden la entrada en vigor del tratado entre el país que formula la reserva y el que formula la objeción. Una posición que no siempre puede llevar a la solución de la controversia surgida al respecto, salvo en aquellos casos en los que el Estado que formula la reserva opta por retirarla como resultado de las presiones ejercidas por parte de la comunidad internacional ${ }^{22}$.

19 Aunque algunas de ellas fueron posteriormente retiradas, como las reservas formuladas a la Convenio Internacional sobre Eliminación de todas las formas de Discriminación Racial, en concreto a su artículo XXII, que fue retirada en 1999. Publicada en BOE 282 , de 25 de noviembre de 1999. Así como la reserva formulada a la totalidad del artículo IX del Convenio para la prevención y la sanción del delito de genocidio, una reserva que había recibido objeciones y que fue retirada en 2009. Publicada en BOE 297, de 10 de diciembre de 2009.

${ }_{20}$ Vid. El instrumento de Ratificación de 16 de diciembre de 1983 de esta convención, publicado en BOE núm. 69, de 21 de marzo de 1984.

${ }_{21}$ Vid. El instrumento de Ratificación de la convención, publicada en BOE núm. 313, de 31 de diciembre de 1990.

${ }_{22}$ Como hicieran, por ejemplo, Chile o Alemania. Ambos países retiraron sus reservas a la Convención contra la tortura y otros tratos o penas crueles, inhumanos o degradantes, tras recibir objeciones por parte de varios Estados, entre los que se encontraba España. 
En este sentido, cabe destacar que la práctica convencional de Filipinas en relación con las objeciones es bastante escasa. Tanto es así, que en el desarrollo de este estudio no se ha observado objeción alguna por parte de Filipinas. Sin embargo en el caso de España sí es muy significativo el número de objeciones formuladas a aquellas reservas que considera incompatibles con el objeto y fin del tratado, una tendencia acorde con la de otros países europeos $^{23}$.

Es más, España ha formulado objeciones a multitud de reservas de otros países en aquellos tratados de Derechos Humanos de los que este país es parte. De hecho, existe alguna objeción por parte de España en la práctica totalidad de los convenios que han sido analizados.

En algún caso se ha llegado a observar la elevada cifra de siete objeciones de España en un mismo tratado multilateral, concretamente a reservas formuladas a la Convención sobre la eliminación de todas las formas de discriminación contra la mujer. En otros casos el número de objeciones formuladas ha sido algo inferior, como en el caso del Pacto internacional de derechos civiles y políticos, respecto del cual se han formulado cuatro objeciones, o la Convención contra la tortura y otros tratos o penas crueles, inhumanos o degradantes, que consta de tres objeciones por parte de España a algunas de las reservas que han sido formuladas. Así pues, en este estudio se han analizado un total de 19 objeciones por parte de este $\operatorname{Estado}^{24}$, formuladas principalmente a reservas de países de Oriente $\mathrm{Medio}^{25}$, aunque no son las únicas reservas que han recibido objeciones por parte de España ${ }^{26}$.

Sin embargo, resulta llamativo sobre el particular el hecho de que una convención con un alcance internacional tan amplio como es la Convención sobre derechos del niño, y por consiguiente con un elevado número de

${ }^{23}$ Se trata de «la práctica internacional más reciente de una tendencia contraria al silencio mostrada por la mayoría de los Estados partes en los tratados sobre derechos humanos, demostrativa de una postura más firme respecto de unas reservas que ponen en peligro la integridad y los objetivos fundamentales de los tratados normativos». García Rico 2010: 237.

${ }^{24}$ Por lo que respecta a los demás tratados internacionales examinados, en la mayoría de ellos hay sólo una objeción por parte de España a alguna reserva. En el caso de la Convención sobre los derechos de las personas con discapacidad, son dos las objeciones formuladas.

${ }_{25}$ Siete objeciones en total: dos objeciones a reservas de Arabia Saudita, dos a reservas de Qatar, y una objeción respectivamente a reservas por parte de la República Árabe Siria, Emiratos Árabes Unidos y Omán.

${ }^{26}$ También hay objeciones a reservas formuladas por parte de países del Sudeste Asiático, como Tailandia y Brunei Darussalam. Igualmente encontramos objeciones a reservas de: Estados Unidos de América (en concreto, a dos reservas), Pakistán (tres objeciones), las Maldivas, Botswana, Corea (dos objeciones) y Bangladesh. 
reservas, no ha recibido objeción alguna por parte de España. Asimismo, este país tampoco ha formulado ninguna objeción a las reservas o declaraciones formuladas a la Convención Internacional para la protección de todas las personas contra las desapariciones forzadas ni al Convenio constitutivo del fondo para el desarrollo de los pueblos indígenas de América Latina y el Caribe. Aunque no sorprende en estos casos la ausencia de objeciones por parte de España ya que, como ya se puso de manifiesto en páginas precedentes de este estudio, se trata de dos convenios con un reducido alcance internacional y que, por consiguiente, cuentan con un mínimo número de reservas ${ }^{27}$.

No obstante, llegados a este punto cabe preguntarse la importancia de las relaciones bilaterales de los Estados en el contexto de la protección de los Derechos Humanos ${ }^{28}$, máxime teniendo en cuenta que una objeción por parte de un Estado a una reserva no impide la entrada en vigor del tratado entre el Estado objetante y el Estado autor de la reserva, salvo que así se estipule expresamente.

Finalmente cabe hacer referencia a aquellas disposiciones contenidas en el propio texto convencional de determinados tratados internacionales, destinadas a asumir la competencia de un Comité, previa declaración de reconocimiento. Una disposición de estas características se encuentra contenida en el texto de los siguientes tratados: la Convención contra la tortura y otros tratos o penas crueles, inhumanos o degradantes, en la Convención Internacional para la protección de todas las personas contra las desapariciones forzadas $^{29}$, en el Pacto internacional de derechos civiles y políticos y en el Convenio Internacional sobre Eliminación de todas las formas de Discriminación Racial. Todos estos Comités han sido reconocidos por España, mientras que Filipinas sólo ha reconocido la competencia del Comité del Pacto internacional de derechos civiles y políticos, si bien hemos de decir que la declaración por la que un país decide someterse a la supervisión de un Comité se puede hacer en cualquier momento. No obstante, cabe destacar que en la mayoría de los casos estudiados la competencia de estos Comités

${ }^{27}$ En el caso de la Convención Internacional para la protección de todas las personas contra las desapariciones forzadas, sólo cinco de sus 57 Estados partes han formulado alguna reserva o declaración. Por otro lado, como ya se dijo con carácter previo, al Convenio constitutivo del fondo para el desarrollo de los pueblos indígenas de América Latina y el Caribe sólo se le ha formulado una declaración por parte de uno de sus 23 Estados partes. Por tanto, no es de extrañar en estos casos la ausencia de objeciones por parte de otros países.

${ }_{28}$ Como bien apunta Marín Aís 2015: 20.

${ }^{29}$ Filipinas no ha hecho una declaración de reconocimiento del Comité, puesto que no es parte de este convenio. 
no viene determinada en el texto de los tratados, sino en los denominados protocolos facultativos ${ }^{30}$ a los que a continuación nos referimos.

\section{Práctica relativa a los protocolos facultativos}

Por último, se ha considerado conveniente ocuparse del análisis de los protocolos facultativos de estas convenciones, en los que se establece la creación de Comités como órganos de control en la aplicación de estos convenios $^{31}$. En este caso la tendencia apunta a un menor número de Estados partes, en comparación con el número de países que han ratificado el correspondiente tratado multilateral del que emana cada uno de estos instrumentos internacionales. Por consiguiente, las declaraciones y las reservas en este caso no serían tan abundantes, aunque seguimos encontrando importantes objeciones a algunas de ellas ${ }^{32}$.

En este sentido, podemos destacar algunos protocolos que cuentan con un elevado número de ratificaciones, como es el caso del Protocolo Facultativo del Pacto Internacional de Derechos civiles y políticos, del que son partes 116 Estados. Sin embargo, el otro protocolo derivado de este convenio: el Segundo Protocolo Facultativo del Pacto Internacional de Derechos civiles y políticos destinado a abolir la pena de muerte, tiene en la actualidad un número de Estados partes sensiblemente menor que el anterior, en concreto 84 países. Además, el menor número de ratificaciones de este protocolo ha dado lugar sólo a cinco reservas ${ }^{33}$ y dos declaraciones,

${ }^{30}$ De cuya ratificación por parte del Estado depende el reconocimiento de la competencia del Comité.

${ }^{31}$ Se trata de órganos de vigilancia «con el mandato de supervisar la aplicación de los tratados que los crean. (...) un control para asegurar que los Estados cumplen con las obligaciones previstas en los tratados internacionales que reconocen derechos humanos». Alija Fernández y Bonet Pérez 2016: 22.

32 Pues, al igual que ocurre con los tratados internacionales, también encontramos algún caso en el que se han retirado determinadas reservas como respuesta a las presiones ejercidas por otros Estados partes del protocolo, como hiciera El Salvador respecto de su reserva al Protocolo facultativo a la Convención sobre los derechos de las personas con discapacidad ante la objeción de Alemania. El Estado alemán advertía de que la ambigüedad de la reserva formulada por El Salvador, cuyo contenido genérico e impreciso hacía una referencia en sentido amplio a su normativa interna, era contraria al objeto y fin del tratado. Vid., https://treaties.un.org/Pages/ViewDetails.aspx?src=TREATY\&mtdsg_ no $=$ IV-15-a\&chapter $=4 \&$ clang $=$ en $\# 4$

33 Además de tres reservas que fueron posteriormente retiradas por parte de: Chipre, Malta y España. Asimismo, cabe destacar que se han formulado 15 objeciones a estas reservas, una de ellas por parte de España, de las cuales 14 son a la reserva de El Salvador y una a la de Brasil. Reservas y objeciones disponibles en: https://treaties.un.org/Pages/ ViewDetails.aspx?src=TREATY\&mtdsg_no=IV-12\&chapter=4\&clang=_en\#9 
frente a la multitud de reservas y de declaraciones interpretativas formuladas al primer protocolo ${ }^{34}$.

Por otro lado, el Protocolo Facultativo de la Convención sobre la eliminación de todas las formas de discriminación contra la mujer también tiene un gran alcance internacional, específicamente son 109 sus Estados partes, a pesar de lo cual sólo se han formulado seis declaraciones, ninguna de las cuales ha recibido objeción alguna por parte de otro Estado. En este caso las cifras contrastan con los datos correspondientes a la convención internacional de la que deriva este protocolo facultativo, puesto que la Convención sobre la eliminación de todas las formas de discriminación contra la mujer cuenta con un elevadísimo número de reservas y declaraciones interpretativas, así como de objeciones a las mismas.

Algo similar sucede con el Protocolo Facultativo a la Convención sobre los derechos de las personas con discapacidad, del que son partes 92 Estados, entre los que no se encuentra Filipinas. Sin embargo, sólo se han formulado tres declaraciones, por parte de: Azerbaiyán, la República Árabe Siria y Turquía ${ }^{35}$.

Por lo que respecta a los protocolos facultativos derivados de la Convención sobre los Derechos del niño, en concreto: sobre la participación de niños en conflictos $\operatorname{armados}^{36}$ y el Protocolo relativo a la venta de niños, la prostitución infantil y la utilización de niños en la pornografía; el número de Estados partes es muy superior al de otros protocolos, en concreto 166 y 173 partes respectivamente ${ }^{37}$. No obstante, los datos obtenidos en este sentido no

${ }^{34}$ Una de ellas formulada por España, concretamente:

"El Gobierno Español se adhiere al Protocolo facultativo del Pacto Internacional de derechos civiles y políticos, interpretando el artículo 5, párrafo 2, de este protocolo, en el sentido de que el Comité de Derechos Humanos no considerara ninguna comunicación de un individuo a menos que se haya cerciorado de que el mismo asunto no ha sido sometido o no lo esté siendo a otro procedimiento de examen o arreglo internacionales». Vid, Instrumento de Adhesión de España al Protocolo facultativo, BOE 79, de 2 de abril de 1985.

Además, dos de las reservas formuladas a este Protocolo han recibido objeciones, en concreto la formulada por la República de Trinidad y Tobago y por Guyana (una de las objeciones formuladas por parte de España).

35 A la que se han formulado tres objeciones, por parte de Austria, Chipre y Grecia.

${ }_{36}$ Al que tanto España como Filipinas formularon una declaración, en ambos casos estableciendo que la edad mínima de reclutamiento voluntario en sus Fuerzas Armadas es de 18 años.

${ }^{37}$ Entre los que se encuentran Filipinas y España. Este último ha formulado una objeción en cada uno de estos Protocolos: en el primer caso a la reserva formulada por Omán y en el segundo a la reserva formulada por Qatar.

Es interesante que los protocolos facultativos de la Convención sobre los Derechos del niño permiten a todos los Estados ratificarlos o adherirse a ellos, con independencia de 
resultan especialmente sorprendentes teniendo en cuenta que se trata de protocolos cuyo objeto no es la creación de un Comité como órgano de control, sino de ámbitos de protección concretos derivados de una convención con un gran alcance internacional ${ }^{38}$. Sin embargo, el número de Estados partes de estos protocolos contrasta con los escasos 34 países $^{39}$ que son partes del tercer protocolo facultativo que deriva de esta convención, el Protocolo facultativo de la Convención sobre los Derechos del Niño relativo a un procedimiento de comunicaciones, cuyo objeto es reconocer la competencia del Comité de los derechos del niño así como establecer un sistema de comunicaciones y un procedimiento de investigación.

Por su parte, entre los protocolos que cuentan con un menor número de ratificaciones a la fecha de redacción de este artículo, se encuentran el Protocolo Facultativo del Pacto Internacional de Derechos Económicos, Sociales y Culturales, con el escaso número de 22 Estados partes ${ }^{40}$, y el Protocolo facultativo a la Convención contra la tortura y otros tratos o penas crueles, inhumanos o degradantes, con 83 Estados partes. En este último caso, Filipinas hizo la siguiente declaración:

«In accordance with Part V, Article 24 of the Optional Protocol to the Convention against Torture and Other Cruel, Inhuman, or Degrading Treatment or Punishment, the Republic of the Philippines hereby declares the postponement of the implementation of its obligations under Part III of the Optional Protocol, specifically Article 11 (1)(a) on the visitations by the Subcommittee on Prevention to places referred to in Article 4 and for them to make recommendations to States Parties concerning the protection of persons deprived of their liberty against torture and other cruel, inhuman or degrading treatment or punishment.. ${ }^{41}$

Como se puede observar, la práctica de Filipinas y España en lo que respecta al número de reservas o declaraciones formuladas a estos Protocolos facultativos guarda similitud con la práctica de estos Estados relativa a los textos convencionales. Del mismo modo, el número de objeciones se puede equiparar en ambos casos puesto que, si bien seguimos sin encontrar

que sean partes o no de la Convención. Por ejemplo, Estados Unidos es parte de estos protocolos pero no ha ratificado la Convención, simplemente procedió a su firma en 1995.

${ }^{38}$ De hecho, de todos los convenios sobre Derechos Humanos examinados en el desarrollo de este trabajo, la Convención sobre los Derechos del niño es la que más ratificaciones ha recibido por parte de los Estados.

39 Entre ellos España. Sin embargo, a día de hoy, Filipinas no es parte de este protocolo, al igual que otros muchos Estados que han ratificado o se han adherido a la Convención sobre los derechos del niño.

40 Entre los que no se encuentra Filipinas.

${ }^{41}$ Disponible en https://treaties.un.org/Pages/ViewDetails.aspx?src=TREATY\& mtdsg_no=IV-9-b\&chapter $=4 \&$ clang $=$ en 
objeciones por parte de Filipinas a ninguna reserva formulada a estos protocolos por parte de otro Estado, en el caso de España encontramos un total de cuatro objeciones: un descenso lógico en el número de objeciones si tenemos en cuenta que el número de reservas formuladas a los protocolos facultativos es inferior que en el caso de las convenciones.

\section{REFLEXIONES FINALES}

De todo lo expuesto se deduce que la práctica convencional de Filipinas en lo que respecta a los tratados destinados a la protección de Derechos Humanos es muy homogénea: prácticamente todos los convenios analizados en el desarrollo de este estudio han sido ratificados por Filipinas y apenas se han encontrado reservas por parte de este Estado, lo que representa una práctica conforme a la salvaguardia de los Derechos Humanos contenidos en estos tratados multilaterales de carácter universal. Sin embargo, no se han observado objeciones a reservas formuladas por otros Estados partes, lo que deja entrever una actitud pasiva por parte de este país hacia la protección de la integridad del texto convencional.

Por otro lado, en cuanto a los Comités como organismos de control y supervisión del tratado, hemos de decir que Filipinas no reconoce la competencia de muchos de ellos, lo que contrasta con el elevado número de tratados internacionales del que este Estado es parte.

En el caso de España, su práctica convencional es algo más variada. España es parte de diez de los catorce tratados que han sido analizados, aunque no se caracteriza por formular reservas o declaraciones interpretativas a estos instrumentos convencionales. No obstante, sí destaca el número de objeciones formuladas a aquellas reservas que este Estado considera incompatibles con el objeto y fin del tratado, en aras de preservar el principio de integridad de estos convenios. Asimismo, cabe destacar que España ha aceptado la competencia de los Comités de todos aquellos convenios que han sido objeto de estudio, lo que representa una práctica acorde con la debida protección de los Derechos Humanos contenidos en los tratados de los que este país es parte.

\section{BIBIOGRAFÍA}

Alija Fernández, R.A. y Bonet Pérez, J., El control de los Tratados Internacionales de Derechos Humanos: realidad y límites. Una aproximación a la labor de los órganos de expertos desde la perspectiva de la efectividad de los derechos económicos, sociales y culturales, Atelier (Barcelona, 2016): 22.

GARCÍA RICO, E.M., «Algunas reflexiones en torno a la práctica española sobre reservas a los tratados de Derechos Humanos de ámbito universal», los derechos hu- 
manos en la sociedad internacional del siglo XXI, vol. 2, Colección Escuela Diplomática, $\mathrm{n}^{\circ} 16,(2010): 237$.

Horn, F., Reservations and Interpretative Declarations to Multilateral Treaties, T.M.C. Asser Instituut, (North-Holland, 1988): 514.

Imbert, P.H., Les Réserves aux Traités Multilatéraux, Publications de la Revue Générale de Droit International Public, Editions A. Pedone, (Paris, 1979): 503.

MARín Aís, J.R., «Incompatible reservations with the object and purpose of the Convention on the elimination of all forms of discrimination against women. The case of Islamics States», Revista electrónica de estudios internacionales, $\mathrm{n}^{\circ} 29$ (2015): 20 .

RiQuelme CoRTAdo, R., Las reservas a los tratados. Lagunas y ambigüedades del Régimen de Viena, Universidad de Murcia, Servicio de Publicaciones, (Murcia, 2004): 434. 